\title{
An Hypothesis for CXCL1/CXCR2 Signaling Regulating Neutrophil-Derived Opioid Peptides Involved in Acupuncture for Inflammatory Pain
}

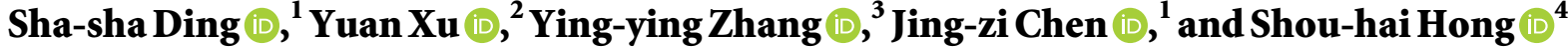 \\ ${ }^{1}$ Acupuncture Physiotherapy Department, Rehabilitation Department, Tianjin Nankai Hospital, Tianjin, China \\ ${ }^{2}$ Acupuncture Research Center, School of Acupuncture and Massage, Tianjin University of Traditional Chinese Medicine, \\ Tianjin, China \\ ${ }^{3}$ Zhejiang Chinese Medical University, Hangzhou, China \\ ${ }^{4}$ Acupuncture Department, Zhejiang Provincial Hospital of TCM, Hangzhou, China
}

Correspondence should be addressed to Shou-hai Hong; hongshouhai@163.com

Received 25 November 2020; Revised 11 April 2021; Accepted 13 April 2021; Published 21 April 2021

Academic Editor: Mohammad Hashem Hashempur

Copyright ( 2021 Sha-sha Ding et al. This is an open access article distributed under the Creative Commons Attribution License, which permits unrestricted use, distribution, and reproduction in any medium, provided the original work is properly cited.

\begin{abstract}
Increasing evidences demonstrate that acupuncture is effective in treating inflammatory pain. Recent studies have found that peripheral endogenous opioid peptides in the area of inflammation are involved in acupuncture-treating inflammatory pain. However, the source of endogenous opioid peptides in local area of inflammation and the mechanism of acupuncture regulating these opioid peptides remain unclear. Studies have demonstrated that neutrophils infiltrated in the inflamed tissue contain and release opioid peptides. Chemokine (C-X-C motif) ligand 1 (CXCL1) is one of the key neutrophil chemokines and can promote the blood neutrophil recruitment to the area of inflammation. In our previous experiments, we found that acupuncture could alleviate inflammatory pain and significantly increase the concentration of chemokine CXCL1 in the blood of rats with inflammatory pain. So we suppose that increased concentration of CXCL1 by acupuncture could activate the blood opioidcontaining neutrophils via its main receptor chemokine receptor type 2 (CXCR2) and promote them recruit to the inflamed tissue to release opioid peptides, participating in the analgesic effect of acupuncture.
\end{abstract}

\section{Background}

At present, there are a large number of patients suffering from pain in clinical practice. Opioid peptides are the potent drugs for the treatment of pain, but their clinical use is limited by detrimental side effects such as respiratory depression, addiction, sedation, and constipation. Therefore, augmenting endogenous opioid actions and selectively targeting peripheral opioid receptors are a new promising approach for opioid analgesia [1]. Numerous studies have found that inflammation could trigger the migration of opioid-containing immunocytes to the inflamed tissue, causing the release of opioid peptides. Numbers of opioid receptors expressed in the sensory nerve fibers in peripheral tissues are significantly increased under inflammatory conditions, and activation of the peripheral opioid receptors can produce a significant analgesic effect without central and gastrointestinal side effects [2-4]. More importantly, evidences have demonstrated the use of peripheral opioid agonists for the prolonged treatment of inflammatory pain has not induced peripheral tolerance, which has important implications for treating inflammatory pain. Therefore, researching the peripheral opioid receptors as new targets for inflammatory pain has a very promising prospect [3-5].

Despite advances in therapies, many patients often continue to deal with ongoing pain symptoms which are not fully controlled by the conventional medicine, so the patients frequently turn to complementary and alternative medicine (such as acupuncture, herbs, and meditation) [6-8]. Many patients with pain, including some children, 
have used complementary and alternative medicine, which is effective in reducing pain, reducing the need for analgesics, and reducing side effects [9]. As an important part of traditional Chinese medicine, acupuncture has been applied in 183 countries and areas in the world. It is widely used in a variety of diseases, especially in the treatment of various kinds of pain. Many studies have shown that acupuncture has broad application prospects due to its unique advantages for the treatment of pain and depression associated with pain $[10,11]$.

\section{Supporting Evidence}

2.1. Peripheral Endogenous Opioid Receptors in the Inflamed Tissue Are Involved in Acupuncture Analgesia. Studies have demonstrated that peripheral endogenous opioid peptides and their receptors in the inflamed tissue were increased after acupuncture [12], and the anti-inflammatory pain effect of acupuncture could be reversed by intraplantar (i.pl.) injection of nonselective peripheral opioid receptor antagonist (naloxone methiodide) and selective antagonists against $\mu, \delta$, or $\kappa$ opioid receptor in the inflamed paw [13-15], whereas acupuncture analgesia could not be blocked by intraperitoneal injection of naloxone methiodide [13] or intravenous injection of selective antagonists [14]. It suggests that endogenous peripheral opioid receptors are involved in acupuncture analgesia.

\subsection{Immunocytes Infiltrated in the Inflamed Tissue Express} and Release Opioid Peptides, Having Significant Analgesic Effects. Neutrophils Are the Most Important Source of Opioids. When the body is under condition of inflammation, immunocytes infiltrate and release numerous inflammatory mediators, causing pain and hyperalgesia in the inflamed tissue [16]. However, recent studies have indicated that immunocytes which infiltrate in the inflamed tissue also release analgesic mediators to relieve pain, including antiinflammatory cytokines and opioid peptides, and the latter is also called peripheral immune cell-derived opioid peptides. Interaction of peripheral opioid peptides with the opioid receptors in the inflamed tissue can effectively reduce pain [2-4].

Peripheral immune cell-derived opioid peptides are mainly derived from leukocytes. Researchers have found that all leukocytes express opioid peptides (endorphin, dynorphin, and enkephalin) (Figure 1) [17], in which the neutrophils account for the largest proportion. Furthermore, inflammation could trigger the opioid-containing leukocytes migrating to the inflamed tissue to release opioid peptides involved in the peripheral intrinsic analgesia mechanism $[3,18]$.

Peripheral immune cell-derived opioid peptide analgesia has some advantages and characteristics as follows: (1) without detrimental side effects of central endogenous opioid, such as nausea, addiction, pain tolerance, and respiratory depression $[3,19,20]$; (2) it may have promising clinical application prospects in the treatment of chronic inflammatory pain. Studies have showed that, in early stages

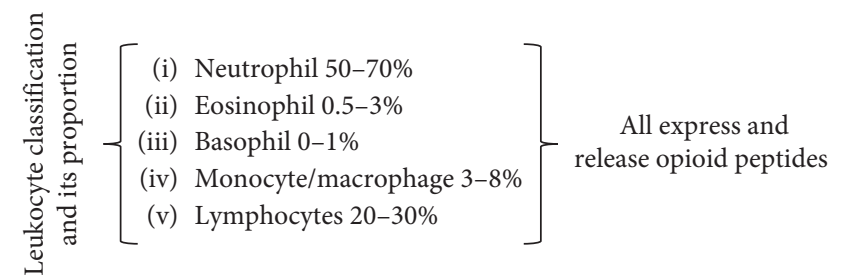

FIgURE 1: Classification and proportion of opioid-containing leukocytes.

of inflammation ( $6 \mathrm{~h}$ after inflammation), both peripheral and central opioid receptors are involved in the antinociceptive effect [21], whereas in later stages (4-6 days after inflammation), endogenous analgesia is mediated exclusively by peripheral opioid and its receptors [19, 22]. However, due to the limitations of experimental studies, pharmacological techniques, or other aspects, in peripheral endogenous opioid peptides for treating inflammatory pain, there are still some challenges to recruit more opioid peptide-containing immune cells to the inflammation site and to efficiently promote the release of immune cell-derived opioid peptides and the synthesis of opioid receptors in inflammation site [21, 23].

\subsection{Chemokine CXCL1 Promotes the Recruitment of Immune} Cells (Especially Neutrophils) to the Inflamed Tissue. Chemokine (C-X-C motif) ligand 1 (CXCL1) is a C-X-C chemokine family member containing a Glu-Leu-Arg (ELR) motif at its amino terminus. It is one of the major chemoattractants for neutrophils. CXCL1, also known as neutrophil chemokine 1, belongs to the CXC family of chemokines [24]. CXCL1 can activate and promote leukocyte recruitment to inflamed tissue via chemokine receptor type 2 (CXCR2) receptor (the main receptor of CXCL1) located on the surface of leukocytes [24-26]. Previous studies considered that leukocyte recruitment was one of the key factors causing inflammatory pain, but recent studies have proved that the migration of leukocytes to the inflamed tissue triggered by CXCL1 binding to CXCR2 receptor does not produce pain $[27,28]$; reversely, the pain is increased significantly after inhibiting the recruitment of leukocytes (especially neutrophils) [27]. Further studies have found that CXCL1 can induce leukocytes to release opioid peptides in inflamed tissue to produce peripheral analgesia and antiinflammatory effects [3].

\subsection{Chemokine CXCL1 in the Blood of Rats Mediates the Anti-} Inflammatory Pain Effect of Acupuncture. Our previous experiments discovered that acupuncture at Zusanli acupoint could alleviate inflammatory pain and significantly upregulate the expression of CXCL1 in the serum of rats with adjuvant-induced arthritis (AIA) (Figure 2) [29]. After neutralizing CXCL1 in the blood with specific antibodies, the anti-inflammatory pain effect of acupuncture was significantly decreased, and intravenous injection of CXCL1 recombinant protein could simulate the anti-inflammatory pain effect of acupuncture. These results indicated that 


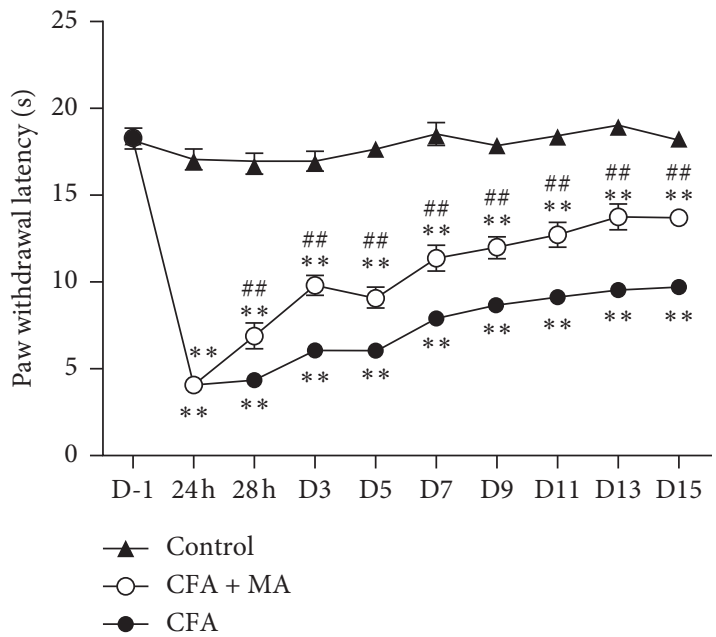

(a)

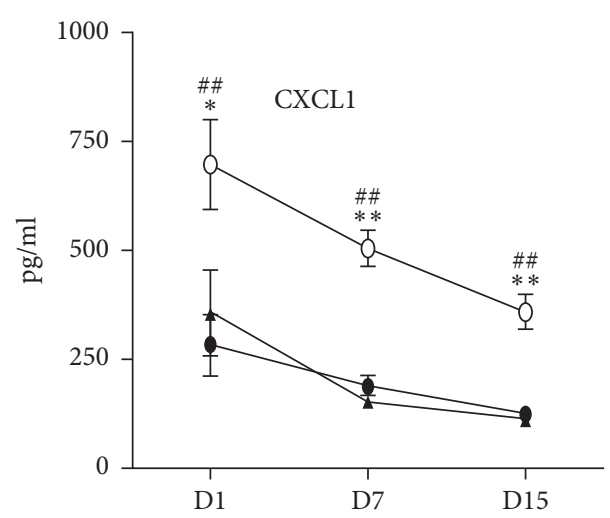

(b)

FIgURE 2: Acupuncture at Zusanli acupoint could alleviate inflammatory pain and significantly upregulate the expression of CXCL1 in the serum of rats with AIA (CXCL1: chemokine (C-X-C motif) ligand 1; AIA: adjuvant-induced arthritis).

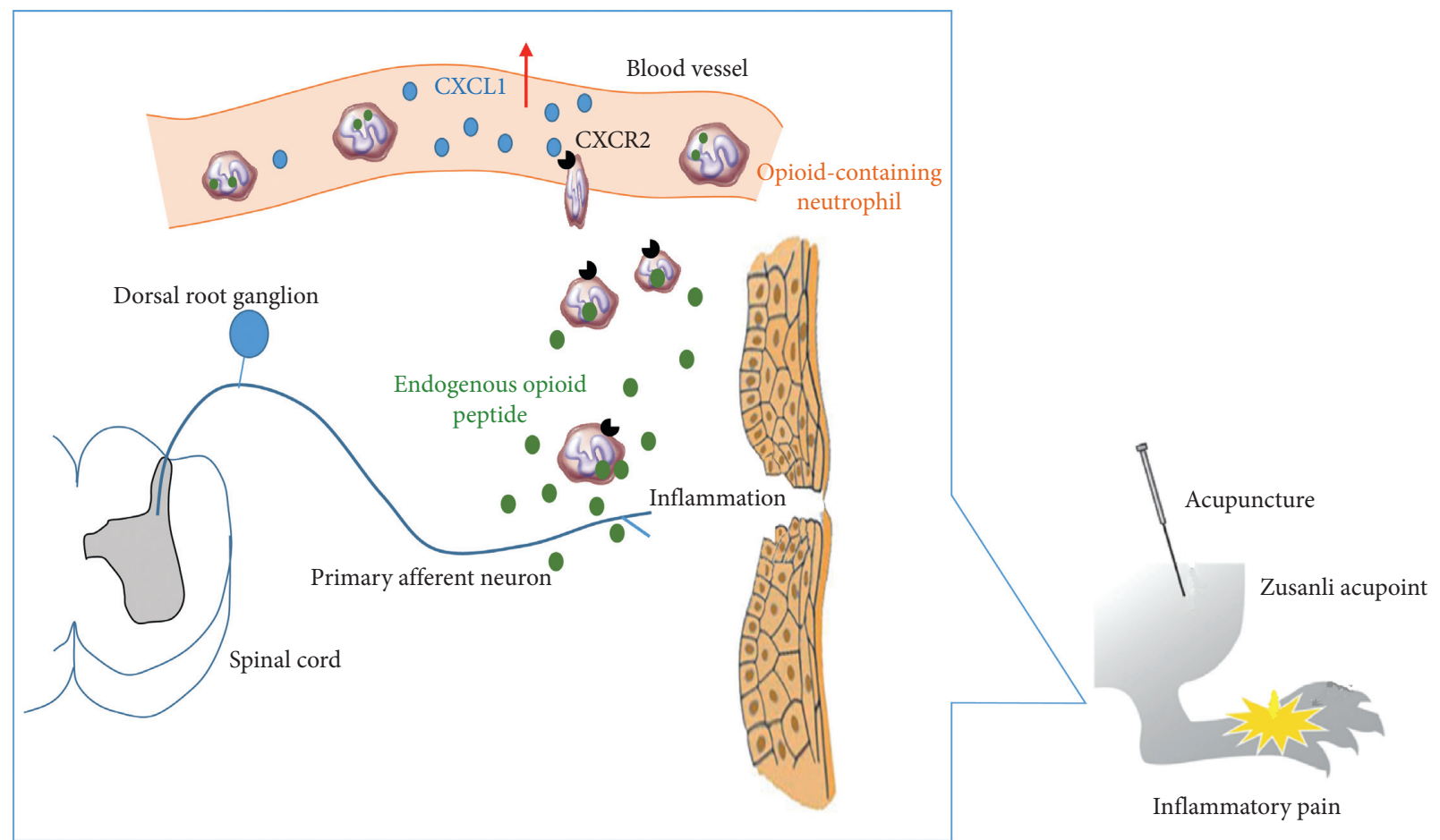

FIGURE 3: Schematic hypothesis of CXCL1/CXCR2 signaling regulating neutrophil-derived opioids in acupuncture for inflammatory pain (CXCL1: chemokine (C-X-C motif) ligand 1; CXCR2: chemokine receptor type 2).

CXCL1 in the blood probably mediated the anti-inflammatory pain effect of acupuncture (data not yet published).

In summary, we speculate that acupuncture can significantly increase the concentration of chemokine CXCL1 in the blood of AIA rats and promote CXCL1 to activate opioid-containing neutrophils in the blood via CXCR2 receptor and trigger the recruitment of neutrophils to the inflamed tissue to release the opioid peptides, participating in the anti-inflammatory pain effect of acupuncture (Figure 3).

\section{Conclusion}

As the peripheral opioid peptides are without central side effects, the development of peripheral opioid agonists has attracted more attention. There are kinds of peripheral opioid agonists in the current [20]. For peripheral endogenous opioid analgesia of inflammatory pain, little studies pay attention on how to increase the release of immune cell-derived opioids in the inflamed tissue. Our hypothesis could provide some ideas for the research. There are still some limitations: 
this is a preliminary hypothesis formed on the basis of our previous research findings and current published results, and it needs some experiments to verify this hypothesis.

\section{Conflicts of Interest}

The authors declare that there are no conflicts of interest.

\section{Acknowledgments}

This research was supported by the National Natural Science Foundation of China (Grant nos. 81804182 and 81603677) and Zhejiang Provincial Natural Science Foundation of China (Grant no. LY20H270008).

\section{References}

[1] C. Stein, "Current opinion in supportive and palliative care 2020 section: opioid analgesia: recent developments," Current Opinion in Supportive Palliative Care, vol. 14, 2020.

[2] S. Gonzalez-Rodriguez, M. A. Quadir, S. Gupta et al., "Polyglycerol-opioid conjugate produces analgesia devoid of side effects," ELIFE, vol. 6, 2017.

[3] K. S. Iwaszkiewicz, J. J. Schneider, and S. Hua, "Targeting peripheral opioid receptors to promote analgesic and antiinflammatory actions," Frontiers in Pharmacology, vol. 4, p. 132, 2013.

[4] S. Hua, "Neuroimmune interaction in the regulation of peripheral opioid-mediated analgesia in inflammation," Frontiers in Immunology, vol. 7, p. 293, 2016.

[5] V. G. Del, V. Spahn, and C. Stein, "Novel opioid analgesics and side effects," ACS Chemical Neuroscience, vol. 8, pp. 1638-1640, 2017.

[6] B. A. Bauer, J. C. Tilburt, A. Sood, G.-X. Li, and S.-H. Wang, "Complementary and alternative medicine therapies for chronic pain," Chinese Journal of Integrative Medicine, vol. 22, no. 6, pp. 403-411, 2016.

[7] M. Anvari, H. Dortaj, B. Hashemibeni, and M. Pourentezari, "Application of some herbal medicine used for the treatment of osteoarthritis and chondrogenesis," Traditional and Integrative Medicine, vol. 5, pp. 126-149, 2020.

[8] M. Maghbool, T. Khosravi, S. Vojdani et al., "The effects of eugenol nanoemulsion on pain caused by arteriovenous fistula cannulation in hemodialysis patients: a randomized double-blinded controlled cross-over trial," Complementary Therapies in Medicine, vol. 52, Article ID 102440, 2020.

[9] L. Chen and A. Michalsen, "Management of chronic pain using complementary and integrative medicine," BMJ, vol. 357, Article ID j1284, 2017.

[10] A. J. Vickers, E. A. Vertosick, G. Lewith et al., "Acupuncture for chronic pain: update of an individual patient data metaanalysis," The Journal of Pain, vol. 19, no. 5, pp. 455-474, 2018.

[11] B. Yan, S. Zhu, Y. Wang, G. Da, and G. Tian, "Effect of acupuncture on chronic pain with depression: a systematic review," Evidence-Based Complementary and Alternative Medicine, vol. 2020, Article ID 7479459, 10 pages, 2020.

[12] H. J. Chen, M. Li, and L. Chen, "Mechanism of acupuncture analgesia and endogenous cannabinoid system in integumentum commune," Medical Journal of Wuhan University, vol. 4, pp. 564-568, 2011.

[13] G. G. Zhang, C. Yu, W. Lee, L. Lao, K. Ren, and B. M. Berman, "Involvement of peripheral opioid mechanisms in electroacupuncture analgesia," Explore, vol. 1, no. 5, pp. 365-371, 2005.
[14] R. Taguchi, T. Taguchi, and H. Kitakoji, "Involvement of peripheral opioid receptors in electroacupuncture analgesia for carrageenan-induced hyperalgesia," Brain Research, vol. 1355, pp. 97-103, 2010.

[15] Y. Wang, R. Gehringer, S. A. Mousa, D. Hackel, A. Brack, and H. L. Rittner, "CXCL10 controls inflammatory pain via opioid peptide-containing macrophages in electroacupuncture," PLOS One, vol. 9, Article ID e94696, 2014.

[16] R.-R. Ji, A. Chamessian, and Y.-Q. Zhang, "Pain regulation by non-neuronal cells and inflammation," Science, vol. 354, no. 6312 , pp. 572-577, 2016.

[17] C. Jacobi and C. Stein, "Inflammatory-linked changes in CpG island methylation of three opioid peptide genes in a rat model for pain," PLOS One, vol. 13, Article ID e191698, 2018.

[18] Y.-L. Jiang, X.-F. He, Y.-F. Shen et al., "Analgesic roles of peripheral intrinsic met-enkephalin and dynorphin A in longlasting inflammatory pain induced by complete Freund's adjuvant in rats," Experimental and Therapeutic Medicine, vol. 9, no. 6, pp. 2344-2348, 2015.

[19] D. Labuz, S. Berger, S. A. Mousa et al., "Peripheral antinociceptive effects of exogenous and immune cell-derived endomorphins in prolonged inflammatory pain," Journal of Neuroscience, vol. 26, no. 16, pp. 4350-4358, 2006.

[20] V. Martínez and R. Abalo, "Peripherally acting opioid analgesics and peripherally-induced analgesia," Behavioural Pharmacology, vol. 31, no. 2, pp. 136-158, 2020.

[21] S. Hua and P. J. Cabot, "Mechanisms of peripheral immunecell-mediated analgesia in inflammation: clinical and therapeutic implications," Trends in Pharmacological Sciences, vol. 31, no. 9, pp. 427-433, 2010.

[22] D. Labuz, S. A. Mousa, M. Schäfer, C. Stein, and H. Machelska, "Relative contribution of peripheral versus central opioid receptors to antinociception," Brain Research, vol. 1160 , pp. 30-38, 2007.

[23] S. Hua and P. J. Cabot, "Targeted nanoparticles that mimic immune cells in pain control inducing analgesic and antiinflammatory actions: a potential novel treatment of acute and chronic pain conditions," Pain Physician, vol. 16, no. 1, pp. E199-E216, 2013.

[24] R. L. Silva, A. H. Lopes, R. M. Guimarães, and T. M. Cunha, "CXCL1/CXCR2 signaling in pathological pain: role in peripheral and central sensitization," Neurobiology of Disease, vol. 105, pp. 109-116, 2017.

[25] R.-R. Ji, Z.-Z. Xu, and Y.-J. Gao, "Emerging targets in neuroinflammation-driven chronic pain," Nature Reviews Drug Discovery, vol. 13, no. 7, pp. 533-548, 2014.

[26] Z.-J. Zhang, D.-L. Cao, X. Zhang, R.-R. Ji, and Y.-J. Gao, "Chemokine contribution to neuropathic pain: respective induction of CXCL1 and CXCR2 in spinal cord astrocytes and neurons," Pain, vol. 154, no. 10, pp. 2185-2197, 2013.

[27] A. Brack, H. L. Rittner, H. Machelska et al., "Control of inflammatory pain by chemokine-mediated recruitment of opioid-containing polymorphonuclear cells," Pain, vol. 112, no. 3, pp. 229-238, 2004.

[28] H. L. Rittner, S. A. Mousa, D. Labuz, K. Beschmann, M. Schäfer, and A. Brack, "Selective local PMN recruitment by CXCL1 or CXCL2/3 injection does not cause inflammatory pain," Journal of Leukocyte Biology, vol. 79, no. 5, pp. 1022-1032, 2006.

[29] Y. Xu, S. Hong, X. Zhao et al., "Acupuncture alleviates rheumatoid arthritis by immune-network modulation," The American Journal of Chinese Medicine, vol. 46, no. 05, pp. 997-1019, 2018. 